Journal of

Epilepsy and

Clinical

Neurophysiology

J Epilepsy Clin Neurophysiol 2006; 12(3):161-167

\title{
História da Epilepsia: Um Ponto de Vista Epistemológico*
}

\author{
Marleide da Mota Gomes** \\ Instituto de Neurologia Deolindo Couto - UFRJ
}

\begin{abstract}
RESUMO
Introdução: A história da epilepsia estuda a evolução do conhecimento médico com as suas contradições e incrementos aqui abordados eurocentristicamente. A literatura relacionada é rica e extensa. Metodologia: Revisão narrativa baseada em fatos históricos sobre epilepsia em perspectiva epistemológica. Objetivos: Apresentar uma breve resenha da evolução do conhecimento e de fatos no decorrer de quase 2300 anos desde Hipócrates até meados do século XIX, na virada para a epileptologia moderna que tem como símbolo, John Hughlings Jackson (1834-1911). Mencionar, da era pré-moderna, as hipóteses sobre a origem das crises epilépticas (CE) no organismo humano e suas causas e os conseqüentes procedimentos terapêuticos. Arrolar, dos anos subseqüentes ao mestre inglês, aspectos fundamentais da epileptologia moderna através dos seus Centros precursores, personalidades e fatos mais marcantes: terapia medicamentosa e cirúrgica, diagnóstico neurofisiológico e o retorno à abordagem integrada biopsicossocial patrocinada pela Campanha Global Contra Epilepsia, Saindo das sombras. Considerar: personalidades com/ou apenas hipoteticamente com epilepsia; literatura feita por escritores com epilepsia ou expectadores das CE de pessoas com epilepsia ou conhecedores de relatos secundários delas; livros médicos e periódicos precursores sobre epilepsia. Conclusão: A história da epilepsia consegue fazer uma resenha da evolução do conhecimento médico o que pode ser feito for fatos científicos e suas interfaces socioculturais. Ela demonstra, por exemplo, a importância histórica do método dedutivo-hipotético na elucidação da fisiopatogenia da epilepsia, magistralmente feita por John Hughlings Jackson, ao lado do mais usualmente usado nas Ciências, o indutivo.
\end{abstract}

Unitermos: epilepsia, história, epistemologia

\begin{abstract}
History of epilepsy: a epistemologic point of view

Introduction: The history of the epilepsy studies the medical knowledge with their contradictions and increments, here presented in a European point of view. The related literature is rich and extensive. Methodology: Narrative review based on epilepsy historical facts in an epistemologic perspective. Objectives: To present an overview of the evolution of the knowledge and of facts in elapsing of almost 2300 years from Hippocrates to middles of the XIX century, in the turn point of the modern epileptology that has as symbol, John Hughlings Jackson (1834-1911). To mention, of the pre-modern era, the hypotheses on the seizure origin in the human organism and related causes and the consequent therapeutic procedures. To enroll, after the subsequent years to the English master, fundamental aspects of the modern epileptology through their pioneer Centers, personalities and more outstanding facts: surgical and drug therapies, neurophysiological diagnosis and the return to the integrated bio-psycho-social approach sponsored by the Global Campaign Against Epilepsy, Out of the shadows. To consider: personalities with/or just hypothetically with epilepsy; literature done by writers with epilepsy or who saw patient's seizures or who read secondary reports about these patients; precursor medical book or periodics. Conclusion: The epilepsy history manages to do a review of the medical evolution of the knowledge that can be done by means of scientific facts and socio-cultural interfaces. It demonstrates, for instance, the historical importance of the deductive-hypothetical method in the elucidation of the epilepsy physiopathogeny, masterfully done by John Hughlings Jackson, besides the more usually carried out in the Sciences, the inductive.
\end{abstract}

Key words: epilepsy, history, epistemology.

\footnotetext{
* Trabalho parcialmente apresentado no XXXI Congresso Brasileiro de Neurologia realizado em Bento Gonçalves, de 21 a 25 de junho de 2006 e aqui exposto como uma homenagem aos 200 anos da Faculdade de Medicina da UFRJ criada pelo príncipe regente D. João em 1808.

** Pós-Graduação do Programa de Psiquiatria e Saúde Mental da UFRJ. Programa de Epilepsia do Instituto de Neurologia Deolindo Couto/UFRJ. Received June. 27, 2006; accepted Aug. 10, 2006.
} 


\section{INTRODUÇÃO}

Poucas doenças chamaram tanta atenção e geraram tanto debate quanto a epilepsia. A sua literatura é extensa e precursora das neurociências, da diferenciação explícita entre práticas culturais religiosas, mágicas e científicas ${ }^{(1)}$. Ela demonstra como a exposição à história dos cuidados médicos é importante pedagogicamente. Os estudiosos da medicina reconhecem que todos os conhecimentos médicos são sujeitos à mudança e adquiridos em contextos específicos. Isso pode ser inferido ao longo do presente texto, a partir de uma revisão narrativa, que procura fazer uso da filosofia das neurociências para entender a construção do conhecimento sobre a epilepsia, o que se pensa sobre ela e porque se pensa desta forma. Assim, a autora além de expor fatos históricos principais também procura explorar criticamente a construção do conhecimento sobre a epilepsia ao longo do tempo, especialmente por John Hughlings Jackson (1834-1911).

\section{MARCOS HISTÓRICOS ATÉ O INÍCIO DA EPILEPTOLOGIA MODERNA}

\section{Etiologia}

As crenças predominam na história da epilepsia. As pessoas com epilepsia (PCE) da Roma antiga eram evitadas por medo de contágio ${ }^{(2)}$. Na Idade Média, elas foram perseguidas como bruxas ${ }^{(2)}$. Em 1494, foi lançado o manual de caçar bruxas, Malleus maleficarum, escrito por dois frades dominicanos vinculados à famigerada Inquisição Católica. Nesse tratado, a presença de crises epilépticas (CE) era uma característica de feitiçaria ${ }^{(2)}$. A orientação do mencionado tratado levou à perseguição, tortura e morte a mais de 100.000 mulheres $^{(2)}$, conclui-se que várias delas eram PCE. Na primeira metade do século passado nos EUA, essas pessoas eram rotuladas como desviantes e o seu matrimônio e reprodução eram restringidas através de legislação e médicos eugenicistas, como Gordon Lennox (1884-1960)(2,3). Existem também relatos de conversão religiosa relacionada temporalmente à $\mathrm{CE}$, assim como o acometimento de epilepsia de vários líderes religiosos ${ }^{(2)}$. A visão da epilepsia como devida a influências ocultas ou más teve partidários até mesmo na Medicina durante os tempos antigos. Conseqüentemente, foram prescritos tratamentos mágicos ou religiosos, algumas práticas que persistem até hoje em parte da população leiga. No Renascimento, houve a tentativa de se ver a epilepsia como uma manifestação de doença física em lugar de uma mais obscura. No entanto, foi durante o Iluminismo que a epilepsia começou a ser considerada de forma mais moderna, com a ajuda de avanços da anatomia, patologia, química, farmácia e fisiologia(2). A evolução do conhecimento sobre o transtorno é pontificado por nomes como Hipócrates, Galeno, Arateus, Avicena, Paracelsus, Willis,
Boerhaave e Tissot. Nas seções seguintes, mencionamos outros personagens e aquisições nesta saga para entender este complexo sintomatológico e os cuidados às $\mathrm{CE}$ e às PCE. O Corpus Hipocraticum, conceitos médicos de Hipócrates e de sua escola, com abordagem holística, se fez valer até meados dos anos de 1700. Esses conceitos se apoiavam em variados aforismos sendo que alguns ainda norteiam a Medicina e a ética médica. Vários dos textos a seguir apresentados e transcritos por Pearce (1998)(4) demonstram como ao longo do tempo teorias fisiopatogênicas das CE se somaram escalarmente. Andréas Vesalius (1514-1564) reconheceu a epilepsia focal. Brown-Séquard (1858) teve um papel importante na teoria da irritabilidade de nervo aferente periférico, sendo a medula o componente central do mecanismo reflexo. Robert Bentley Todd acreditou que as CE eram conseqüentes ao acometimento inicial dos lobos hemisféricos, logo seguido pelo corpo quadrigêmino, sendo a intensidade da perturbação dependendente do comprometimento da medula oblongata e da espinha dorsal. Nothnagel gerou a hipótese do "centro convulsivo" adjacente ao do da respiração. Hammond no seu tratado On the diseases of the nervous system (1871) pensou que a localização da epilepsia era na medula e as lesões no córtex a excitariam para produzir a convulsão. No entanto, várias teorias patofisiológicas da epilepsia identificaram corretamente o cérebro como a origem do problema, mas enfatizando causas incorretas. Galeno (130-200) deduziu que a epilepsia era um transtorno do cérebro devido ao acúmulo de humores espessos, sendo que Paracelsus reconhecia uma ebulição para cima dos espíritos vitais (spiritus animalis). Thomas Willis (16211675) indicou que a predisposição do cérebro para ter CE era hereditária ou adquirida. De acordo com BrownSéquard, o vasospasmo cerebral reflexo, em lugar de congestão venosa cerebral, seria a causa da perda de consciência. Jacobus Schroeder van der Kolk (1797-1862) realizou autópsias e caracterizou: "dilatação das veias que apareceram cheias de sangue no córtex, medula e espinha dorsal." Assim, no decorrer desses 2.400 anos passamos da hipótese do supernatural, com denominações de "doença sagrada" e "lunáticos", para uma natural orientada por Hipócrates e texto védico Charaka Samhita(5) até as idéias mais modernas. Como poderemos concluir mais a seguir, na seção sobre as Escolas precursoras, as concepções mais modernas sobre as raízes do transtorno começaram a ocorrer apenas a partir dos séculos XVIII e XIX.

\section{Terapêutica}

relato das tentativas malsucedidas terapêuticas é extenso. Muitas não somente eram aleatórias e ineficazes como poderiam também ser cruéis, a considerar algumas como: consumo de sangue de ser humano recentemente 
morto, pó de crânio humano, digitalis ou nitrato de prata, além de sangria, purgação, emese, diurese, sudorese e recomendação para exercer ou coibir atividade sexual ou trepanação craniana, p. ex. ${ }^{(2)}$. Como as CE estavam muitas vezes relacionadas a causas sobrenaturais, a elas se recorriam para neutralizá-las: amuletos e santos tais como São Valentim. Conclui-se que esses tratamentos eram usualmente ineficazes, mas comumente baseados nas hipóteses fisiopatogênicas em curso das CE recorrentes.

\section{EPILEPSIA EM PERSONALIDADES DESTACADAS E NA ARTE}

Ressalta-se que a maioria das PCE era vista com preconceito, mas alguns tiveram sucesso e ficaram famosas, sendo várias estadistas, santos, cientistas, artistas, havendo notório viés masculino ${ }^{(2)}$. No entanto, Hughes $(2005)^{(6)}$ alerta sobre o exagero de imputar a várias personalidades a condição de epiléptico tendo ao contrário outros problemas de saúde como os que mais freqüentemente também confundem o diagnóstico na prática clínica como as crises psicogênicas, de angústia, nervosas, de medo, de agitação, de fraqueza ou de abstinência ao álcool. Em alguns casos, o autor não encontrou nenhuma evidência de qualquer sintoma episódico. Ele considera pouco provável ou mesmo inadequado o diagnóstico de epilepsia, ou apenas de epilepsia, para Pitágoras (582-500 a.C), Aristóteles (384-322 a.C), Aníbal (247-183 a.C), Dante Alighiere (1265-1321), Joana D'Arc (1412-1431), Leonardo da Vinci (1452-1519), por exemplo. No Brasil, algumas personalidades brasileiras de mais a menos famosas eram notoriamente epilépticas, como Dom Pedro I (1798-1834)(7), ícone da independência do Brasil, célebre pela sua impulsividade e sexualidade, com epilepsia de incidência familiar, que gerou muitos filhos, a independência do Brasil e a reconquista de Portugal para os liberais; Antônio Moreira César (1850-1897) ${ }^{(8)}$, desastrado e feroz comandante da 3a tropa contra os jagunços de Antonio Conselheiro em Canudos e Joaquim Maria Machado de Assis (1839-1908) ${ }^{(9)}$, o mundialmente consagrado elegante e discreto escritor brasileiro. A comentar que a Faculdade de Medicina da UFRJ foi fundada pela família real portuguesa, dentre outros marcos desenvolvimentistas do Brasil por ela lançados. Essa família tinha entre os seus familiares, muitos sofredores de epilepsia de natureza familiar, dentre eles o já mencionado Pedro I. Quanto à literatura, ela é pródiga nos relatos a PCE e ou CE. Wolf $(2006)^{(10)}$ lembra que textos literários são uma parte importante da história cultural de muitos campos de medicina. Segundo esse autor, relatos sobre epilepsia e sua semiologia são freqüentemente descritas: por escritores que sofreram CE, sendo que alguns provêem relatos perspicazes notáveis e modernos sobre os sintomas subjetivos e experiências ictal e periictal; outros que observam com objetividade que pode ser clínica de CE em parentes íntimos ou estranhos fortuitos; e o último grupo de escritores que relatam crises não observadas por eles próprios mas reconhecidas de outros tipos de testemunhos. O livro mais famoso, a Bíblia, no seu novo testamento, como no evangelho segundo São Marcos - 9:14-29, apresenta um pai que relata as CE de seu filho. $\mathrm{O}$ mais prolífico dos escritores a escrever sobre a epilepsia foi Fiodor Mikhailovitch Dostoievski (18211881), ele próprio uma PCE. Em contrapartida, um dos mais discretos a lidar com o transtorno e também PCE, foi José Maria Machado de Assis (1839-1908). Algumas passagens de sua obra é mais textual quanto a essa relação, como as sugestivas crises de automatismo, outras bem menos. Essas descrições se atém ao estilo literário sutil do escritor e têm seu significado apenas sugerido, mas nunca ostentado. Wolf (2006) (10) lembra que Machado de Assis fez uso literário de sua experiência com CE parciais complexas, possivelmente por epilepsia do lobo temporal direito $^{(9)}$ em uma época que a sua semiologia era virtualmente desconhecida.

\section{LIVROS MÉDICOS SOBRE EPILEPSIA E PERIÓDICOS PRECURSORES}

A epilepsia induziu a literatura médica a privilegiar esta área do conhecimento humano. Existe mesmo um livro clássico $(1945,1971)^{(11)}$ sobre a sua história produzido por Owsei Temkin (1902-2002), que aborda dos primórdios da história da medicina ocidental até John Hugling Jackson. Várias obras ressaltam a importância da epilepsia em estudos pioneiro. Dentre elas, destacamos algumas a seguir. Do conjunto de setenta escritos atribuído à escola de Hipócrates denominado de Corpus hippocraticum ${ }^{(12)}$, apenas um é referente a um complexo sintomatológico: Da Doença Sagrada. Nele se lê: "Parece-me que ela não é mais divina ou sagrada do que qualquer outra doença; mas ela tem causa natural como as outras doenças”. Várias das obras a seguir apresentadas favoreceram/sistematizaram o conhecimento da época e a classificação da epilepsia ${ }^{(13,14)}$ : Samuel-Auguste Tissot, Traité de l'epilepsie (1770); Jean Baumes, Traité des convulsions de l'enfance (1787 e 1805); Theodore Herpin (postumamente), Des accès incomplets d'épilepsie, (1867); William Richard Gowers, Epilepsy and other chronic convulsive diseases; their cause etc. (1881); Russel R Reynolds, Epilepsy: its symptoms, treatment, and relation to other chronic convulsive diseases (1861); Frederic e Erna Gibbs publicaram Atlas of electroencephalography (1941, 1951); William Gordon Lennox e Margaret Lennox, Epilepsy and Related Disorders (1960).

Ressaltamos dois periódicos, um internacional e outro nacional. Epilepsia, órgão oficial da International League against Epilepsy (ILAE). O seu $1^{\circ}$ volume foi edi- 
tado em 1909. No Brasil, a Liga Brasileira de Epilepsia (LBE) lançou pioneiramente um órgão de divulgação de uma subespecialidade neurológica: Boletim da LBE, 1978-1981, após Jornal da LBE (1988) que se transformou em Brazilian Journal of Epilepsy and Clinical Neurophysiology até a designação atual de Journal of Epilepsy and Clinical Neurophysiology.

\section{MEDICINA MODERNA E EPILEPTOLOGIA}

\section{Escolas precursoras}

Mencionaremos os berços da moderna epileptologia, a começar pelo Neurological Institute - Londres, Hospital for the "paralyzed and epileptic" (1857). Outros a reconhecer são: Harvard University e Illinois University; Neurological Institute of Montreal; Hospital La Timone de Marselha e Hospital Saint-Anne de Paris. Do primeiro Centro, três neurologistas ingleses se destacaram no início da era moderna da epilepsia: além de Jackson, Russell Reynolds e William Richard Gowers (1845-1915). Jackson favoreceu a localização funcional cerebral e a organização hierárquica do sistema nervoso. Isso foi um grande avanço conceitual que tornou possível o desenvolvimento da neurociência moderna no século XIX. Na base da reconstrução histórica sobre a epilepsia, a heurística de Jackson gerou uma transformação adicional que favoreceu o conhecimento da medicina atual. Ela pôde responder aos fenômenos da epileptogenicidade que seriam comprovados mais tarde em bases experimentais. A definição de Jackson (1873) de epilepsia como "uma descarga súbita, excessiva e rápida da substância cinzenta" ainda hoje é acertada, precedendo os estudos sobre excitabilidade elétrica cerebral de David Ferrier (1874), na Inglaterra, e Gustav Fritsch e Eduard Hitzig (1870), na Alemanha ${ }^{(5,15)}$. Jackson postulou o mecanismo da psicose pós-epiléptica considerando que o cérebro é um sistema hierárquico, com a consciência no seu mais alto nível. Em vigência da CE, ela seria inibida permitindo a expressão dos níveis mais primitivos $^{(15)}$. Isso conduziu à teoria da: evolução-dissolução (na primeira referente ao níveis, na segunda, o nível mais alto seria mais vulnerabilizado e o mais inferior, retido); sintomas positivo-negativos, isto é lesões transitórias levariam tanto a fenômenos negativos, como a paralisia, quanto positivos (irritativos), como a epilepsia. Assim, esses fenômenos seriam liberados por perda da inibição ou modulação dos níveis funcionais mais altos ${ }^{(2,15,16)}$. A idéia sobre o "dreamy state", de "diplopia mental", que levou ao conceito de epilepsia do lobo temporal também tem essa base $^{(15)}$. Os princípios evolucionistas de Herbert Spencer influenciaram as teorias de Jackson sobre a organização cerebral $^{(15,16)}$. Lembra-se que a Ciência moderna caminha principalmente sobre os preceitos do racionalismo e empirismo e na Medicina há uma tendência ao uso simultâ- neo e complementar dessas duas tendências epistemológicas. Jackson usou esses recursos nas suas teorias e o racionalismo fundamentou a sua teoria fisiopatogênico da epilepsia, a partir do método hipotético-dedutivo ${ }^{(17)}$. A ressaltar na escola francesa, Theodore Herpin (1799. 1865), que se aproximou do requinte de conhecimentos sobre epilepsia de Jackson, segundo Eadie $(2002)^{(14)}$. No seu livro, Des accès Incomplets d'épilepsie, publicado postumamente (1867) ele relata uma gama pormenorizada e extensa de possíveis manifestações não convulsivas das CE(14). Para Eadie (2002) ${ }^{(14)}$, ele pode ser considerado "um dos grandes documentos na história da epileptologia". Porém, o médico não notou a presença de CE de ausência em quaisquer dos seus 300 pacientes $^{(14)}$. A palavra "epilepsia" se referia às CE tônico-clônica generalizadas, sendo que as outras manifestações eram consideradas como manifestações incompletas de epilepsia ${ }^{(14)}$. Herpin reconheceu que todos as $\mathrm{CE}$, completas ou incompletas, se originam no mesmo lugar do cérebro o que pode ser depreendido a partir de um relato de caso ${ }^{(14)}$. As suas observações e interpretação parecem ter precedido o desenvolvimento independente de Jackson sobre conceitos semelhantes, mas a exploração intelectual mais extensa do inglês das implicações das suas observações lhe fez uma figura mais importante do que Herpin na história da epileptologia ${ }^{(14)}$. No entanto, a obra de Herpin parece ter influenciado o trabalho de Jackson, como lembra Eadie $(2002)^{(14)}$. No outro lado do Canal da Mancha, vicejava a "doença" chamada de histero-epilepsia por Jean Martin Charcot $(1825-1893)^{(18)}$ que combinava transtorno da mente e do cérebro, da histeria e da epilepsia(2). $\mathrm{Na}$ Salpêtrière, o local de nascimento e epicentro de neurologia moderna, Charcot encontrou as PCE ao lado dos oligofrênicos, pacientes com sífilis crônica, criminosos insanos e histéricos. Sendo que esses últimos, vulneráveis à sugestão e persuasão, começaram a imitar os ataques epilépticos que testemunhavam repetidamente. Assim, a epileptologia francesa era derivada principalmente de PCE de asilos e hospitais de pacientes mentalmente crônicos ${ }^{(14)}$. A diferenciar, ao contrário, a clientela de Herpin mais representativa da comunidade ${ }^{(14)}$. Charcot reconheceu a importância de Jackson e é dele a designação da epilepsia Bravais-Jacksoniana, inicialmente vinculada apenas à Bravais, por conta da sua tese $(1824)^{(14)}$. Lembra-se que Jackson designava esse tipo de epilepsia a "convulsões epileptiformes"(15). Voltando à escola inglesa pioneira, lembramos de Gowers claramente favorável a ser no córtex a gênese das CE: “... todos os fenômenos das crises da epilepsia idiopática podem ser explicados pela descarga da substância cinzenta; que a hipótese de espasmo vascular é tão desnecessária quanto não está comprovada; ... que epilepsia é uma doença da substância cinzenta, e não tem nenhum local uniforme”. Gowers (1881) classificou epi- 
lepsia como grand mal, petit mal e histeróide ${ }^{(19)}$. J. Russell Reynolds (1861) classificou as convulsões associadas com um transtorno estrutural do sistema nervosa de epilepsia sintomática, as associadas com condições fora do sistema nervoso central (insuficiência renal, por exemplo) de epilepsia simpática ${ }^{(19)}$. $\mathrm{Na}$ Harvard University e Illinois University, o EEG se desenvolveu e teve importante papel na clínica da epilepsia: início dos anos de 1930, 1 canal EEG; 1935, 3 canais, com Albert Grass e Frederic Andrews Gibbs (1903-1992), em Harvard; 1944, Erna e Frederic se mudaram para a Universidade de Illinois. Gibbs também é considerado o primeiro a enfatizar a associação da epilepsia do lobo temporal a transtornos comportamentais ${ }^{(2)}$. Lennox, da Escola de Harvard, "o pai" do tratamento moderno da epilepsia, ficou quatro anos como um médico missionário na China de lá retornando para Boston quando sua filha mais nova desenvolveu epilepsia ${ }^{(3,20)}$. Ele trabalhou com Frederic Gibbs e estudou circulação e metabolismo cerebral, enquanto localizava a doença em milhares de famílias e mantinha registros cuidadosos de muitos gêmeos, tentando determinar se epilepsia era uma característica herdada. Ele foi presidente da ILAE de 1935-1946, co-editor e depois editor da Revista Epilepsia. Ele organizou a American Epilepsy League, Committee for Public Understanding of Epilepsy, foi chefe da Divisão de Epilepsia Children's Medical Center de Boston onde estabeleceu um programa de treinamento para médicos em epilepsia. Lennox escreveu, com a sua filha Margaret um importante livro sobre epilepsia que contém a descrição da síndrome de Lennox-Gastaut. No Neurological Institute de Montreal, pontificaram Herbert Henri Jasper (19061999), Wilder Penfield (1891-1976) e Theodore Brown Rasmussem (1910-2002). A partir de 1939, Jasper colaborou com Penfield. $O$ trabalho deles proveu bases para localização de foco de crises epilépticas pelo EEG. A estimulação cortical direta favoreceu a definição da anatomia funcional do cérebro humano. Nesse Centro, foi reconhecida a esclerose mesial temporal e epilepsia por Murray Falconer e o grupo dele e William Feindel e a conseqüente base cirúrgica mais comum da epilepsia focal tratável. Rasmussen sucedeu Penfield como diretor do Instituto Neurológico de Montreal. Realizou muitas cirurgias para epilepsia. Em Marselha, no Hospital La Timone, Henri Gastaut (1915-1995) foi consagrado como grande epileptologista pelas suas bases anatomopatológicas, neurofisiológicas em aliança ao seu intelecto arguto $^{(21)}$. Ele foi chefe dos Laboratórios de Neurobiologia do Hospital de Marselha (1953) e diretor do centro regional para epilepsia em 1960(21). De 1973 a 1984, assumiu a cadeira de neurofisiologia clínica ${ }^{(21)}$. Ele promoveu o estudo do EEG e epilepsia (1947-1987)(21). Foi secretário geral e presidente da ILAE com várias contribuições ao desenvolvimento dos seus capítulos ${ }^{(21)}$, a destacar o brasileiro presidido por
Paulo Niemeyer. Com a sua esposa e parceira nos estudos de EEG, Ivete, reconheceu padrões diversos do EEG tais como ondas lambda, ritmo mi, ritmo pi, ritmo teta posterior e pontas rolândicas. Ele favoreceu o uso de vários métodos de ativação dessa técnica, fez correlações fisiológicas das parassonias e uso em vários estados de alteração de consciência natural ou artificial (meditação ou biofeedback, por exemplo) ${ }^{(21)}$. Ele valorizou a semiologia da epilepsia e contribuiu com: nosografia, classificação da epilepsia adotada pela ILAE, definição da síndrome HHE (hemiplegia, hemiconvulsão e epilepsia), epilepsia do susto (startle epilepsy), colaboração na definição das síndromes de Lennox-Gastaut e West e epilepsia parcial benigna occipital (forma de Gastaut), associação da epilepsia com o comportamento e diagnóstico diferencial com os eventos não epilépticos ${ }^{(21)}$. A sua abordagem reconhecia o valor da integração biopsicossocial das $\mathrm{PCE}^{(21)}$. Ele também procurou entender possíveis vínculos entre epilepsia e genialidade artística. Não desconsiderava a importância do estudo da arte e literatura. Em Paris, no Hospital SaintAnne o trabalho consagrou o neurocirurgião Jean Tailarach e o neurofisiologista clínico Jean Bancaud (1921-1993). O início da era moderna da Epileptologia no Brasil veio com Paulo Niemeyer (1914-2004) que foi: fundador da

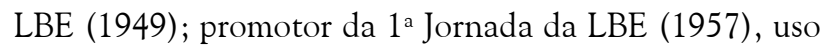
pioneiro da eletrocorticografia e exploração eletrográfica com eletrodos implantados no Brasil; inventor da amigdalahipocampectomia na epilepsia do lobo temporal e descritor da anormalidade elétrica neocortical pós-cirúrgica ${ }^{(22)}$. Helio de Paiva Bello, Ilustre discípulo de Henri Gastaut, da "Escola de Marselha", foi o genial parceiro de Paulo Niemeyer ${ }^{(23)}$ nas mencionadas conquistas e líder de uma grande e clássica escola de eletroencefalografia brasileira.

\section{Neurofisiologia clínica}

Após os descobrimentos iniciais da Eletroencefalografia em seres humanos por Hans Berger (1873-1941) houve sua aplicação clínica na epilepsia, nos anos de 1930(24): 1934, Fisher e Lowenback foram os primeiros a demonstrar pontas epileptiformes; 1935, Gibbs, Davis e Lennox descreveram ponta-onda interictal e padrões $3 \mathrm{~Hz}$ do complexo ponta-onda, na ausência epiléptica dando alento ao campo da EEG clínica; 1936, Gibbs e Jasper definiram as pontas interictais como assinatura focal da epilepsia. A localização de descargas epilépticas pela EEG ampliou as possibilidades de tratamento cirúrgico, mais amplamente disponível a partir dos anos de 1950. A análise quantitativa do EEG nos anos de 1940 e 1950 incrementou a análise temporal e espacial do EEG ${ }^{(25)}$. Mary Brazier (1904-1995) foi a pioneira da utilização da função de coerência e de fase para o estudo da propagação da atividade epiléptica crítica registrada com eletrodos intracranianos $(1968)^{(25)}$. 


\section{Terapêutica medicamentosa e outras}

A idéia de que a irritação focal podia causar CE apoiou o controle das CE por sedativos, primeiro pelos brometos (Charles Locock em 1857) e barbitúricos (Hauptmann em 1912), introduzidos na clínica sem avaliação pré-clínica ${ }^{(26)}$. Mais especificamente, o brometo foi inicialmente usado para sedar mulheres jovens com histeria, após para as com epilepsia até chegar à epilepsia catamenial e às PCE de modo geral ${ }^{(2)}$. A introdução da fenitoína (Merrit e Putnam em 1937) demonstrou que as drogas não sedativas também poderiam ser efetivas no controle dessas crises, sendo que o modelo pré-clínico usado foi a CE promovida por eletrochoque máximo(26). Várias drogas foram experimentadas e aquelas demonstradas eficazes levaram ao estudo de outras da mesma família farmacológica, muitas não referendando a droga protótipo. Conclui-se que algumas dessas drogas usadas foram conseqüentes a achados serendipiticos, como o valproato, ou avaliação experimental de compostos usando modelos de epilepsia ${ }^{(26)}$. O primeiro desses modelos foi para testar o aumento do GABA no cérebro ${ }^{(26)}$. Mais recentemente, o crescimento da compreensão dos mecanismos da epileptogênese levaram a uma base mais racional para essas descobertas, a partir da década de $1980^{(26)}$. Apontamos a terapêutica que teve mais êxito e permaneceu mais tempo no mercado: 1857, Brometo (já há muito desconsiderada no tratamento humano das PCE); 1912, Fenobarbital; 1920, Dieta cetogênica (de uso clínico iniciado nos anos de 1920(27); 1938, Fenitoína ${ }^{(26)}$; 1963, Carbamazepina ${ }^{(26)}$; 1958, Etosuximida(26); 1967, Valproato de sódio(26); 1997, Estimulação nervo vago - CE focal adulto (usada inicialmente no ser humano em 1988 e aprovada pela FDA em $1997^{(27)}$ ). Foram lançadas no mercado várias DAE na década de 1990, a destacar as introduzidas no Brasil tais como gabapentina, lamotrigina, topiramato e oxacarbazepina.

\section{Cirurgia para epilepsia}

A proximidade dos fundamentos da Escola de Jackson e de sua influência direta deram o impulso necessário a Victor Horsley (1857-1916) para os primórdios da cirurgia para epilepsia $^{(28)}$. Em 1886, dos três pacientes operados descritos, dois eram pacientes de Jackson, sendo que em um foi feita lesionectomia e no outro ressecção mais extensa de tumor ${ }^{(15)}$. Outros avanços permitiram a evolução desse procedimento, como o do mapeamento cortical funcional cerebral. Fedor Krause (1857-1937) e Otfrid Föster (1878-1941) foram importantes nesse desenvolvimento ${ }^{(28)}$. Krause introduziu a estimulação farádica para mapear o córtex motor humano ${ }^{(28)}$. Isso foi continuado por Föster, usando anestesia local e assim favorecendo a responsividade do paciente o que possibilitou excisão cortical extensa das cicatrizes corticais sem lesão relevante das áreas eloqüentes $^{(28)}$. No Neurological Institute of Montreal, houve a introdução do EEG complementar à avaliação pré e per-cirúrgica com a colaboração Jasper. Penfield lá militou, mas antes estagiou com Förster (1928), e expandiu os seus conhecimentos do mapeamento das funções cerebrais corticais. Em princípios dos anos 1950, Penfield/equipe (incluindo Falconer que estagiou no Instituto) definiu características anatômicas e patológicas das CE do lobo temporal mesial o que levou ao "procedimento de Montreal" que consiste na lobectomia temporal anterior inclusive com remoção da amígdala e hipocampo ${ }^{(28)}$. Frederic e Erna Gibbs, já transferidos de Boston para Chicago, se associaram ao cirurgião Percival Bailey o que favoreceu a cirurgia da epilepsia do lobo temporal sem lesão aparente, por orientação do EEG expresso na região temporal anterior ${ }^{(28)}$. No entanto, Bailey evitava ressecar nesses casos as estruturas mesiais e inferiores do lobo temporal ${ }^{(28)}$. No Hospital SaintAnne de Paris houve o desenvolvimento da estereoEEG e terminologia de zonas lesional, irritativa e epileptogênica $^{(29)}$. Nesse Centro, pontificaram Tailarach e Bancaud. No final dos anos de 1980 e 1990, o advento da neuroimagem moderna deu ímpeto novo ao tratamento cirúrgico. A ressaltar que ela e o desenvolvimento da genética favoreceram a melhor definição classificatória das crises e síndromes epilépticas.

\section{Abordagem mais ampla}

Tentativas mais sistematizadas de humanizar o tratamento das PCE ocorreram simultaneamente à inauguração do Hospital for the "paralyzed and epileptic" (1857). Nessa época foram estabelecidas colonias para o cuidado e emprego de PCE, em Dianalund na Dinamarca, Chalfont na Inglaterra, Bielefeld - Bethel na Alemanha, Heemstede na Holanda, Sandviakain na Noruega e Centro de Epilepsia em Zurique ${ }^{(5)}$. O mito da epilepsia como uma maldição foi largamente derrotado em culturas modernas, mas o transtorno ainda permanece com um estigma social para muitos pacientes. Conseqüentemente, para real crescimento da epileptologia efetiva houve necessidade de maior atenção às necessidades psicológicas e sociais e qualidade de vida das pessoas com epilepsia, em contrapartida aos avanços tecnológicos. Ressalta-se que eles são de pequena ou nenhuma relevância para 80\% das PCE dos países em desenvolvimento ${ }^{(5)}$. Assim, Organizações se associaram para atendê-las, a saber a ILAE (fundada em 1909), International Bureau for Epilepsy - IBE (fundada em 1961) e a Organização Mundial de Saúde. Essas três instituições estabeleceram a Campanha Global Contra Epilepsia, Saindo das sombras (1997) que tem como objetivo melhorar a prevenção, tratamento, cuidado e serviços para pessoas com epilepsia e elevar consciência pública sobre o transtorno e promover ambiente encorajador no qual as PCE possam viver melhor ${ }^{(5,30)}$. 


\section{CONCLUSÃO}

"Quanto mais distante para trás você puder olhar, mais distante à frente é provável que você veja" Winston Churchill. Essa visão de Jano, deus da mitologia romana, de reconhecer os términos e os começos, de ter a perspectiva do futuro, mas conhecendo o passado é muito útil para o progresso criterioso da medicina. Para o futuro não muito remoto, aventamos a possibilidade para as PCE com CE focais, cirurgias não ablativas, mas aumentativas ou restauradoras, através da liberação focal de DAE, enxerto neuronal, além do uso de células tronco e implantação de estimuladores cerebrais ${ }^{(31)}$. A terapêutica medicamentosa poderá ter avanço significativo ao se conhecerem os mecanismos intrínsicos específicos das CE e formulação de drogas a eles relacionados, por exemplo. No entanto, sempre haverá espaço da idéia hipocrática de primum non nocere e relevância da integração biopsicossocial que norteia a atual campanha promovida pela OMS, ILAE e IBE. O pai da medicina criou uma abordagem holística recomendando o uso de terapias disponíveis com o máximo de parcimônia. Dele também vêm os conceitos ainda vigentes da ética médica a serem considerados em contrapartida à pressão do mercado para o consumo de alternativas tecnológicas novas e dispendiosas, mas não necessariamente efetivas. A ressaltar a importância de metodologias científicas complementares para o crescimento da epileptologia.

\section{REFERENNCIAS}

1. Riggs AJ, Riggs JE. Epilepsy's role in the historical differentiation of religion, magic, and science. Epilepsia. 2005;46(3):452-3.

2. Masia SL, Devinsky O. Epilepsy and behavior: A brief history. Epilepsy Behav. 2000;1(1):27-36.

3. Offen ML. Dealing with "defectives" Foster Kennedy and William Lennox on eugenics. Neurology. 2003;61(5):668-73.

4. Pearce JM. Early accounts of epilepsy: a synopsis. J Neurol Neurosurg Psychiatry. 1998;64(5):679-82.

5. World Health Organization. Epilepsy: historical overview. Fact sheet n. 168. Revised February 2001.

6. Hughes JR. Did all those famous people really have epilepsy? Epilepsy Behav. 2005;6(2):115-39.

7. Macaulay N. D. Pedro: a luta pela liberdade no Brasil e em Portugal, 1798-1834. Trad. André Villalobos. Rio de Janeiro: Record, 1993.

8. Yacubian EM. When epilepsy may have changed history: Antonio Moreira Cesar as the commander of the third expedition in the war of Canudos. Arq Neuropsiquiatr. 2003;61 (2B):503-9.

9. Guerreiro CA. Machado de Assis's epilepsy. Arq Neuropsiquiatr. $1992 ; 50(3): 378-82$.
10. Wolf P. Descriptions of clinical semiology of seizures in literature. Epileptic Disord. 2006;8(1):3-10.

11. Temkin O. The Falling Sickness: A History of Epilepsy from the Greeks to the Beginnings of Modern Neurology. 2. ed. Baltimore: Johns Hopkins University Press; 1971. 467 p.

12. Great book index. An index to online great books in english translation. Hippocrates (about 460-about 377 BC). URL: http:// books.mirror.org/gb.hippocrates.html

13. Gomes MM. Aspectos históricos da epilepsia. Rev. Bras. Neurol 1996;32(5):169-76.

14. Eadie MJ. The epileptology of Theodore Herpin (1799-1865). Epilepsia. 2002;43(10):1256-61.

15. Sengoku A. The contribution of J. H. Jackson to present-day epileptology. Epilepsia. 2002;43(suppl 9):6-8.

16. Swash M. John Hughlings Jackson (1835-1911). J Neurol. 2005; 252(6):745-6.

17. Anderman AAJ. Huglings Jackson's deductive science of the nervous system: a product of his though collective and formative years. Neurology 1997;48:471-81.

18. McHugh PR. Multiple Personality Disorder: Dissociative Identity Disorder. URL: http://www.psycom.net/mchugh.html

19. Epilepsy foundation. Epilepsy: a brief history. URL: http:// www.epilepsyfoundation.org/answerplace/history.cfm

20. William Lennox Obituary. New York Times, 1960-07-23. URL: http://www.laskerfoundation.org/awards/obits/lennoxobit.shtml

21. Dravet C, Roger J. In memoriam, Henri Gastaut, 1915-1995. Epilepsia. 1996;37(4):410-5.

22. Gomes MM, Guerreiro MM. Liga Brasileira de Epilepsia no final do século XX: 50 anos de história. Brazilian Journal of Epilepsy and Clinical Neurophysiology. 1999;5:134-45.

23. Bello H. Homenagem ao Dr. Paulo Niemeyer. J Epilepsy Clin Neurophysiol 2004;10(4):241-4.

24. Swartz BE, Goldensohn ES. Timeline of the history of EEG and associated fields. Electroencephalogr Clin Neurophysiol. 1998; $106(2): 173-6$.

25. Gotman J. A brief history of EEG analysis before the 2001 odyssey. Epileptic Disorders. 2001;3(3):7-10.

26. Klitgaard H. Antiepileptic drug discovery: lessons from the past and future challenges. Acta Neurol Scand. 2005;181(suppl): 68-72.

27. Forcadas-Berdusan MI. Indications and results of nonpharmacological treatments of epilepsies: vagal stimulation, ketogenic diet and gamma rays. Rev Neurol. 2002;35(suppl 1):S144-50.

28. Feindel W. Role of Brain Science in the Evolution of Epilepsy Surgery. URL: http://www.med.mcgill.ca/mjm/v01n02/epilepsy. html

29. Wichert-Ana L, Carlotti Jr CG, Assirati JÁ, Machado HR, Meneghelli UG, Sakamoto AC. Landmarks in the history of epilepsy surgery. J Epilepsy Clinical Neurophysiology. 2003;9(2):77-86.

30. Reynolds EH. The ILAE/IBE/WHO epilepsy global campaign history. International League Against Epilepsy. International Bureau for Epilepsy. Epilepsia. 2002;43(Suppl 6):9-11.

31. Gomes MM. Epilepsia focal refratária: perspectivas. Rev Bras Neurol. 2005;41(2):37-43.

Endereço para correspondência:

Marleide da Mota Gomes

Instituto de Neurologia da UFRJ - Programa de Epilepsia

Av. Venceslau Braz, 95 ,-Botafogo

CEP 22290-140, Rio de Janeiro, RJ, Brasil 